\title{
New approaches in the management of spasticity in multiple sclerosis patients: role of cannabinoids
}

This article was published in the following Dove Press journal:

Therapeutics and Clinical Risk Management

3 February 2010

Number of times this article has been viewed

\section{Paul F Smith \\ Department of Pharmacology and Toxicology, School of Medical Sciences, University of Otago, Dunedin, New Zealand}

\begin{abstract}
Cannabinoids such as Cannabis-based medicinal extracts (CBMEs) are increasingly being used in the treatment of spasticity associated with multiple sclerosis (MS). They have been shown to have a beneficial effect on spasticity; however, this evidence is largely based on subjective rating scales. Objective measurements using the Ashworth scale have tended to show no significant effect; however, the validity of this scale has been questioned. The available clinical trial data suggest that the adverse side effects associated with using CBMEs are generally mild, such as dry mouth, dizziness, somnolence, nausea and intoxication. However, most of these trials were run over a period of months and it is possible that other adverse side effects could develop with long-term use. There may be reason to be concerned about the use of therapeutic cannabinoids by adolescents, people predisposed to psychosis and pregnant women.
\end{abstract}

Keywords: multiple sclerosis, spasticity, cannabinoids, Cannabis

\section{Introduction}

The discovery in the late 1980s that the main psychoactive constituent of the Cannabis sativa plant, $\Delta^{9}$-tetrahydrocannabinol $\left(\Delta^{9}\right.$-THC), acts on a specific cannabinoid receptor in the brain (the 'cannabinoid CB1 receptor'), had an enormous impact on the development of cannabinoids as therapeutic drugs. ${ }^{1}$ Together with the discovery of endogenous ligands ('endocannabinoids') for this and a second subtype of cannabinoid receptor (the ' $\mathrm{CB} 2$ receptor'), such as anandamide (arachidonylethanolamide) and 2-arachidonylglycerol (2-AG), ${ }^{1}$ considerable interest has been generated in the possibility that either natural or synthetic cannabinoids might be used to modulate the endogenous cannabinoid system for therapeutic purposes. One area of clinical application has been the treatment of pain and spasticity in multiple sclerosis (MS). This review will focus specifically on the potential of cannabinoids to relieve spasticity in MS.

Preclinical animal studies suggest that cannabinoids have anti-spastic effects. Since CB1 receptors appear to be located mainly presynaptically and inhibit calcium influx at axon terminals, they are ideally positioned to inhibit the release of classical neurotransmitters such as glutamate. ${ }^{2,3}$ Activation of CB1 receptors also decreases neuronal excitability by activating somatic and dendritic potassium channels. ${ }^{2}$ Using the experimental allergic encephalomyelitis (EAE) mouse model of MS, cannabinoids have been reported to reduce both spasticity and tremor; furthermore, changes in CB1 receptors have been found in the CNS of EAE animals. ${ }^{4-6}$ This has
Correspondence: PF Smith Department of Pharmacology and Toxicology, School of Medical Sciences, University of Otago, Dunedin, New Zealand

Tel +6434795747

Fax +643479 9140

Email paul.smith@stonebow.otago.ac.nz 
led to the proposal that endocannabinoids provide a natural, anti-spastic function in the CNS. ${ }^{7}$

\section{Clinical trial evidence}

Although many patients with MS use Cannabis in order to obtain relief from chronic pain and spasticity, ${ }^{8-10}$ until recently there were few reliable clinical data to demonstrate whether or not it has any effect beyond that of a placebo. The first clinical studies of the effects of $\Delta^{9}$-THC on spasticity in MS were not encouraging. Most of these studies used small sample sizes and $\Delta^{9}$-THC generally had no effect when objective measurements were made. ${ }^{11,12}$ Nonetheless, some patients did report a subjective improvement in symptoms.

The first systematic, placebo-controlled, clinical trial of the effects of $\Delta^{9}$-THC on MS was published by Killestein and colleagues in $2002 .{ }^{13}$ Killestein et al evaluated the effects of oral $\Delta^{9}$-THC $(2 \times 2.5 \mathrm{mg}$ per day; maximum $2 \times 5 \mathrm{mg})$ and a Cannabis sativa plant extract on 16 MS patients with severe spasticity. They quantified spasticity using the Ashworth scale and disability using the Expanded Disability Status Scale (EDSS). Neither the $\Delta^{9}$-THC nor the Cannabis extract significantly reduced spasticity compared to the placebo; however, the Cannabis extract was associated with significantly more adverse side effects. Furthermore, both $\Delta^{9}$-THC and the Cannabis extract worsened the patients' global impression of their condition. The $\Delta^{9}$-THC doses used were low by comparison with later studies; however, they were obviously high enough to produce adverse side effects.

In 2001, the Cannabinoids in Multiple Sclerosis (CAMS) trial was initiated in the UK. This was a blind, placebo-controlled study, coordinated by Derriford Hospital in Plymouth. The trial involved 660 MS patients receiving either $0.25 \mathrm{mg} / \mathrm{kg} \Delta^{9}$-THC (dronabinol, administered orally in $2.5 \mathrm{mg}$ capsules), natural Cannabis oil (Cannador) or a placebo, and measurements of spasticity, pain and tremor were performed. Some patients were also monitored for cognitive side effects. The first of these results was published in 2003 by Zajicek and colleagues. ${ }^{14}$ Patients were treated either with an oral Cannabis extract $(\mathrm{n}=213), \Delta^{9}$-THC $(n=206)$ or placebo $(n=213)$ for 15 weeks and spasticity was quantified using the Ashworth scale. The maximal daily dose was $25 \mathrm{mg}$. The Cannabis extract contained $2.5 \mathrm{mg}$ of $\Delta^{9}$-THC, $1.25 \mathrm{mg}$ of cannabidiol (CBD) and less than $5 \%$ of other cannabinoids. When the placebo was compared with the Cannabis extract, and with $\Delta^{9}$-THC, the mean reduction in the Ashworth scores was not statistically significant ( 0.32 and 0.94 respectively). Nonetheless, there was a significant reduction in patient-reported spasticity. While only $46 \%$ of patients on the placebo reported an improvement in spasticity, $61 \%$ and $60 \%$ of patients receiving the Cannabis extract or $\Delta^{9}$-THC (respectively) reported an improvement. These results were reminiscent of the Killestein and colleagues ${ }^{13}$ study in which patients reported subjective improvement even when objective measurements of spasticity did not indicate any difference. Adverse side effects were generally minor and similar between the placebo, Cannabis extract and $\Delta^{9}$-THC groups. In a 12-month follow-up study of 657 patients, Zajicek and colleagues ${ }^{15}$ reported that $\Delta^{9}$-THC had a significant effect on spasticity according to the Ashworth scale. The mean decrease was $1.82(\mathrm{n}=154)$ compared to the Cannabis extract (mean decrease: $0.1, \mathrm{n}=172$ ) and the placebo groups (mean decrease: $0.23, \mathrm{n}=176$ ). No major adverse effects were reported. It is possible that $\Delta^{9}$-THC had a greater effect on the patients' Ashworth score over time as a result of plasticity in the endocannabinoid system.

Wade and colleagues ${ }^{16}$ performed a double-blind, placebocontrolled, single patient crossover trial involving whole plant extracts of $\Delta^{9}$-THC, CBD or 1:1 CBD: $\Delta^{9}$-THC. The trial included 24 MS patients, 4 patients with spinal cord injury, 1 with brachial plexus damage and another with limb amputation. They reported that $\Delta^{9}$-THC and CBD improved spasticity, but once again there were no significant differences using the Ashworth scale. Three patients experienced transient hypotension and intoxication at the beginning of the treatment.

In a further study, Wade and colleagues ${ }^{17}$ reported the effects of a Cannabis based medicinal extract (CBME, ie, Sativex $^{\circledR}$ ) on 80 patients with MS. The trial was randomized, placebo-controlled and double-blind and the drug was delivered by oromucosal spray $\left(2.7 \mathrm{mg} \Delta^{9}\right.$-THC and $2.5 \mathrm{mg}$ $\mathrm{CBD}$ at each actuation). The patients were allowed to self titrate their doses to achieve the best effect, with maximal doses of $120 \mathrm{mg} \Delta^{9}$-THC and CBD per day. Spasticity was assessed using a visual analogue scale (VAS) and a modified Ashworth scale. At 6 weeks, the VAS scores showed a significant reduction in the patients $(n=39)$ whose primary symptom had been spasticity. However, once again the Ashworth scale data were not significantly different between the CBME and placebo groups. The most common adverse side effects were intoxication and a decrease in lower limb tone and these could be resolved by a reduction in dose. Overall, the results suggested that cannabinoids might be useful for spasticity in specific types of MS patients. ${ }^{18}$

Vaney and colleagues ${ }^{19}$ reported a double blind, placebocontrolled, crossover study of 57 MS patients receiving Cannabis-based oral capsules containing $2.5 \mathrm{mg} \Delta^{9}$-THC and $0.9 \mathrm{mg}$ CBD. The dose escalated, with the maximal daily dose 
of $30 \mathrm{mg} \Delta^{9}$-THC. Efficacy against spasticity was assessed using self report of spasm frequency and the Ashworth Scale. For the 50 patients included in the entire intentionto-treat set, there were no significant differences. However, for 37 patients who received $90 \%$ of their prescribed dose, significant improvements in spasm frequency were observed. Adverse side effects such as dry mouth and blurred vision were slightly more frequent in the active treatment group. In a study primarily directed at lower urinary tract symptoms (LUTS), Brady and colleagues ${ }^{20}$ also reported that the self assessment of spasticity showed a significant improvement, even with a $\Delta^{9}$-THC only extract, and with only minor side effects.

Collin and colleagues ${ }^{21}$ conducted a 6 week, double blind, placebo-controlled trial comparing Sativex and placebo in 199 MS patients. Using a numerical rating scale, they reported a significant decrease in spasticity in the Sativex group compared to placebo. However, they also found that dizziness, blurred vision and impaired attention were more common in the Sativex group.

Wissel and colleagues ${ }^{22}$ studied the effects of the synthetic cannabinoid, nabilone ( $1 \mathrm{mg} /$ day), on pain related to spasticity in a placebo-controlled, crossover trial in 11 patients with chronic upper motor neurone syndrome. They found that pain was significantly reduced; however, spasticity was not, suggesting that even though pain may be spasticity related, spasticity itself is not necessarily reduced by cannabinoids.

In the most recent randomized, double-blind, placebocontrolled trial, Hagenbach and colleagues ${ }^{23}$ evaluated the effects of $\Delta^{9}$-THC on patients with spasticity related to spinal cord injury. They used a spasticity sum score using a modified version of the Ashworth scale and found a significant decrease in spasticity in the $\Delta^{9}$-THC group; however, 15-20 mg $\Delta^{9}$ THC per day were needed to achieve this effect.

Shakespeare and colleagues ${ }^{24}$ have suggested that many commonly used anti-spasticity drugs have also failed to result in statistically significant differences according to the Ashworth scale and that some drugs may produce clinically meaningful changes in spasticity that the Ashworth scale is incapable of detecting.

Another issue is the heterogeneity of the patients studied in some of these clinical trials. The effects of cannabinoids on spasticity caused by MS versus other conditions such as upper motor neurone syndrome, are not necessarily comparable.

\section{Adverse effects of cannabinoids used in clinical trials}

In most cases, the route of administration used has been oral or by some form of aerosol spray, thereby avoiding any adverse effects associated with smoking Cannabis. Most of these studies have been conducted over a period of months and therefore do not necessarily provide information on possible long-term adverse effects of the use of cannabinoids. Nonetheless, they do provide a reasonable estimate of the most likely side effects associated with the use of $\Delta^{9}$-THC or $\Delta^{9}$-THC/CBD over a period of months.

It is well established that $\Delta^{9}$-THC can adversely affect cognitive function with a sufficiently high dose. ${ }^{11}$ The question really is whether such adverse effects occur at the doses of $\Delta^{9}$-THC or a synthetic cannabinoid that would be used therapeutically, especially over the long-term. Although there have been some reports of attentional disturbances, increased reaction time and impaired spatial working memory in clinical trials of the CBMEs, these have been rare and, in general, there have been few reports of cognitive dysfunction. ${ }^{17,19,20,25-27}$ In one of the few systematic studies of the possible adverse side effects of cannabinoids, Aragona and colleagues ${ }^{28}$ conducted an 8-week, randomized, double-blind, placebo-controlled, parallel-group crossover trial of Sativex, using 17 Cannabis-naïve patients with MS. They assessed adverse cognitive events over a 3 -week period using various checklists and the Paced Auditory Serial Additional Test and found no evidence of significant cognitive impairment at the doses used. These results suggest that the regular use of CBMEs for the treatment of MS may not result in significant cognitive impairment, unless there is an interaction with other drugs or the cognitive effects of the disease state.

Another common concern is whether cannabinoid treatment might exacerbate depression or psychosis. Although it was not reported in the most recent clinical trials, a common reason for withdrawal from earlier clinical trials investigating the use of cannabinoids to control pain and nausea, was dysphoria. ${ }^{29,30}$ Nonetheless, while there is evidence that the use of Cannabis itself can increase the risk of psychiatric disorders, ${ }^{31,32}$ especially in adolescents, there is very little evidence that the use of therapeutic cannabinoids can have such effects, at the doses prescribed. In the study by Aragona and colleagues, ${ }^{28}$ they also assessed psychological state over 3 weeks of treatment with Sativex and found no evidence of psychopathology at the doses used. Nonetheless, many MS patients take beta-interferon, which can cause depression as a side effect, and it is conceivable that cannabinoids could potentiate this effect.

There is increasing evidence to suggest that the use of Cannabis during pregnancy can have a lasting negative impact on the fetal nervous system, resulting in deficits in attention, visual analysis and hypothesis testing. ${ }^{33-38}$ While 
there is no evidence at present that cannabinoids used for therapeutic purposes can have this effect, this issue remains unresolved.

Dizziness is a commonly reported side effect in clinical trials of cannabinoids. ${ }^{27,28}$ This has often been attributed to the cardiovascular effects of $\Delta^{9}$-THC; however, vertigo, the sensation of self-motion, is distinct from general dizziness and implies a vestibular cause. In fact, CB1 receptors exist in the brainstem vestibular nucleus and the synthetic CB1 receptor agonist, CP-55,940, has been shown to modulate the firing rate of vestibular nucleus neurons. ${ }^{39,40}$ These results suggest that cannabinoids have the potential to cause dizziness and vertigo by acting on the vestibular nucleus, although they are not likely to be major problems.

\section{Cannabis extracts versus synthetic cannabinoids}

At present, most clinical studies of the effects of cannabinoids on spasticity in MS have used either $\Delta^{9}$-THC or a CBME that contains $\Delta^{9}$-THC, rather than a synthetic cannabinoid receptor agonist. One reason for using CBMEs such as Sativex is that it is claimed that the CBD in the extract has specific effects on spasticity. ${ }^{25,26}$ Nonetheless, $\Delta^{9}$-THC is a weak partial agonist at $\mathrm{CB} 1$ and $\mathrm{CB} 2$ receptors and therefore has less selective effects than a synthetic selective $\mathrm{CB} 1$ receptor agonist. ${ }^{1}$ Pryce and Baker ${ }^{41}$ have in fact reported that it is $\mathrm{CB} 1$ receptors that mediate the anti-spastic effects of cannabinoids. CBD, on the other hand, acts on anandamide hydrolysis/reuptake and cytokines. Of concern is the lack of understanding of the effects of some of the constituents of Cannabis. Whalley et $\mathrm{al}^{42}$ compared standard Cannabis extracts (SCEs) with and without $\Delta^{9}$-THC, and reported that while $\Delta^{9}$-THC depressed evoked depolarizing post-synaptic potentials (PSPs) in rat olfactory cortex neurons, SCEs with and without $\Delta^{9}$-THC could potentiate PSPs. This effect was blocked by the CB1 receptor antagonist, SR141716A. One of the intriguing findings was that the potentiation of PSPs was greater when a $\Delta^{9}$-THC-free SCE was used. The authors speculated that a novel, unknown component in Cannabis may over-ride the decrease in excitatory synaptic transmission caused by $\Delta^{9}-\mathrm{THC}$, and that this constituent may be responsible for the pro-convulsant effects of Cannabis that have sometimes been reported. In another study by Wilkinson et al, ${ }^{43}$ using seizure activity induced in rat piriform cortical brain slices by oxotremorine-M, found that an SCE had a more potent anticonvulsant action than $\Delta^{9}$-THC alone, but that the $\Delta^{9}$-THC-free extract also had anticonvulsant activity. CBD, by contrast, had no anticonvulsant effect.
Even synthetic $\mathrm{CB} 1$ receptor agonists may have variable effects in the CNS, as a result of their action at different $\mathrm{CB}$ receptors and individual variability in $\mathrm{CB}$ receptor expression. ${ }^{44}$

\section{Conclusion}

The therapeutic effects of CBMEs on spasticity that have been reported in clinical trials could best be described as moderate and occurred mainly in subjective scores rather than objective measurements. Therefore, the therapeutic benefit from these drugs may be quite small in return for any adverse side effects that are experienced. Systematic, dose-to-dose comparisons with other anti-spastic drugs such as baclofen have not yet been made; therefore, it is not clear how much therapeutic advantage CBMEs will offer over conventional medications. The clinical trials to date do not indicate serious adverse side effects associated with the use of CBMEs; however, it remains to be seen whether other adverse events appear with long-term use. Nonetheless, it must be noted that the available evidence from clinical trials suggests that many of the adverse effects of cannabinoids decrease with continued use. In this respect, it is important to note that while $\Delta^{9}$-THC acts as a partial agonist at cannabinoid CB1 receptors, most synthetic agonists, such as CP55,940 and WIN 55,212-2, act as full agonists and are therefore likely to have more potent effects. On the basis of currently available data, CBMEs seem unlikely to have serious, long-term adverse effects on cognition or mood. However, there may be reason to be concerned about the effects of CBMEs on adolescents, people predisposed to psychosis, and the fetal nervous system.

\section{Declaration}

The author has no conflict of interest.

\section{References}

1. Pertwee RG. Emerging strategies for exploiting cannabinoid receptor agonists as medicines. Br J Pharmacol. 2009;156(3):397-411.

2. Kreitzer AC, Regehr WG. Retrograde signalling by endocannabinoids. Curr Opin Neurobiol. 2002;12:324-330.

3. Alger BE. Retrograde signalling in the regulation of synaptic transmission: focus on endocannabinoids. Prog Neurobiol. 2002;68: 247-286.

4. Baker D, Pryce G, Croxford JL, et al. Endocannabinoids control spasticity in a multiple sclerosis model. FASEB J. 2001;15:300-302.

5. Baker D, Pryce G, Croxford JL, et al. Cannabinoids control spasticity and tremor in a multiple sclerosis model. Nature. 2000;404:84-87.

6. Berrendero F, Sanchez A, Cabranes A, et al. Changes in cannabinoid (CB1) receptors in striatal and cortical regions of rats with experimental allergic encephalomyelitis, an animal model of multiple sclerosis. Synapse. 2001;41:195-202.

7. Baker D, Pryce G. The endocannabinoid system and multiple sclerosis. Curr Pharm Des. 2008;14(23):2326-2336. 
8. British Medical Association. Therapeutic Uses Of Cannabis. Amsterdam: Harwood Academic Publishers; 1997.

9. Consroe P, Musty R, Rein J, et al. The perceived effects of smoked Cannabis on patients with multiple sclerosis. Eur Neurol. 1997;38: 44-48.

10. Hall WD, Degenhardt LJ, Currow D. Allowing the medical use of Cannabis. Med J Aust. 2001;175:39-40.

11. Smith PF. Cannabinoids for the treatment of multiple sclerosis: No smoke without fire? Exp Rev Neurotherapeutics. 2003;3:327-357.

12. Smith PF. Medicinal Cannabis extracts for the treatment of multiple sclerosis. Curr Opin Investig Drugs. 2004;5:727-730.

13. Killestein J, Hoogervorst ELJ, Reif M, et al. Safety, tolerability and efficacy of orally administered cannabinoids in MS. Neurol. 2002;58:1404-1407.

14. Zajicek J, Fox P, Sanders H, et al. Cannabinoids for the treatment of spasticity and other symptoms related to multiple sclerosis (CAMS study): multicentre, randomised placebo-controlled trial. Lancet. 2003;362:1517-1526.

15. Zajicek JP, Sanders HP, Wright DE, et al. Cannabinoids in multiple sclerosis (CAMS) study: safety and efficacy data for 12 months follow-up. J Neurol Neurosurg Psychiat. 2005;76:1664-1669.

16. Wade DT, Robson P, House H, et al. A preliminary controlled study to determine whether whole-plant Cannabis extracts can improve intractable neurogenic symptoms. Clin Rehabil. 2003;17:21-29.

17. Wade DT, Makela P, Robson P, et al. Do Cannabis-based medicinal extracts have general or specific effects on symptoms in multiple sclerosis? A double-blind, randomized, placebo-controlled study on 160 patients. Multiple Sclerosis. 2004;10:434-441.

18. Killestein J, Polman C. The therapeutic value of cannabinoids in MS: real or imaginary? Multiple Sclerosis. 2004;10:339-340.

19. Vaney C, Heinzel-Gutenbrunner M, Jobin P, et al. Efficacy, safety and tolerability of an orally administered Cannabis extract in the treatment of spasticity in patients with multiple sclerosis: a randomized, double-blind, placebo-controlled, crossover study. Multiple Sclerosis. 2004;10:417-424.

20. Brady CM, DasGupta R, Dalton C, et al. An open-label pilot study of Cannabis based extracts for bladder dysfunction in advanced multiple sclerosis. Multiple Sclerosis. 2004;10:425-433.

21. Collin C, Davies P, Mutiboko IK, et al; Sativex Spasticity in MS Study Group. Randomised controlled trial of Cannabis-based medicine in spasticity caused by multiple sclerosis. Eur J Neurol. 2007;14: 290-296.

22. Wissel J, Haydn T, Muller J, et al. Low dose treatment with the synthetic cannabinoid Nabilone significantly reduces spasticity-related pain. $J$ Neurol. 2006;253(10):1337-1341.

23. Hagenbach U, Luz S, Ghafoor N, et al. The treatment of spasticity with Delta-9-tetrahydrocannabinol in persons with spinal cord injury. Spinal Cord. 2007;45(8):551-562.

24. Shakespeare DT, Boggild, M,Young C. Anti-spasticity agents for multiple sclerosis. Cochrane Database Systematic Reviews. 2000;4: CD001332.

25. Smith PF. Evaluation of Sativex (GW-1000). IDdb3 Current Drugs Database. http://www.iddb3.com 2004:1-19.

26. Smith PF. Sativex (GW-1000). Current Opinion in Investigational Drugs. 2004;5:748-754.
27. Wade DT, Makela PM, House H, et al. Long-term use of a Cannabis-based medicine in the treatment of spasticity and other symptoms in multiple sclerosis. Multiple Sclerosis. 2006;12(5):639-645.

28. Aragona M, Onesti E, Tomassini V, et al. Psychopathological and cognitive effects of therapeutic cannabinoids in multiple sclerosis: a doubleblind, placebo-controlled, crossover study. Clin Neuropharmacol. 2009;32(1):41-47.

29. Campbell FA, Tramer MR, Caroll D, et al. Are cannabinoids an effective and safe treatment option in the management of pain? A qualitative systematic review. Brit Med J. 2001;323:13-16.

30. Tramer MR, Caroll D, Campbell FA, et al. Cannabinoids for control of chemotherapy-induced nausea and vomiting: quantitative systematic review. Brit Med J. 2001;323:16-21.

31. Fergusson D, Poulton R, Smith PF, et al. Cannabis and psychosis. Brit Med J. 2006;332:172-175.

32. Moore THM, Zammit S, Lingford-Hughes A, et al. Cannabis use and risk of psychotic or affective mental health outcomes: A systematic review. Lancet. 2007;370:319-328.

33. Fried PA, Watksinson B. Differential effects on facets of attention in adolescents prenatally exposed to cigarettes and marihuana. Neurotoxicol Teratol. 2001;23:421-430.

34. Fried PA, Smith AM. A literature review of the consequences of prenatal marihuana exposure. An emerging theme of a deficiency in aspects of executive function. Neurotoxicol Teratol. 2001;23:1-11.

35. Fried PA, Watkinson B, Gray R. Differential effects on cognitive functioning in 13- to 16 year-olds prenatally exposed to cigarettes and marihuana. Neurotoxicol Teratol. 2003;25:427-436.

36. Goldschmidt L, Richardson GA, Cornelius MD, et al. Prenatal marijuana and alcohol exposure and academic achievement at age 10. Neurotoxicol Teratol. 2004;26:521-532.

37. Goldschmidt L, Day NL, Richardson GA. Effects of prenatal marijuana exposure on child behavior problems at age 10. Neurotoxicol Teratol. 2000;22:325-336.

38. Smith AM, Fried PA, Hogan MJ, et al. Effects of prenatal marijuana on response inhibition: an fMRI study of young adults. Neurotoxicol Teratol. 2004;26:533-542.

39. Newsham-West D, Darlington CL, Smith PF. Potent effects of a selective cannabinoid receptor agonist on some medial vestibular nucleus neurons. Eur J Pharmacol. 1998;348:R1-R2.

40. Ashton JC, Zheng Y, Liu P, et al. Immunohistochemical characterization and localization of cannabinoid CB1 receptor protein in the rat vestibular nucleus complex and effects of unilateral vestibular deafferentation. Brain Res. 2004;1021:266-273.

41. Pryce G, Baker D. Control of spasticity in a multiple sclerosis model is mediated by CB1, not CB2, cannabinoid receptors. Br J Pharmacol. 2007;150(4):519-525.

42. Whalley BJ, Wilkinson JD, Williamson EM, et al. A novel component of Cannabis extract potentiates excitatory synaptic transmission in rat olfactory cortex in vitro. Neurosci Letts. 2004;365:58-63.

43. Wilkinson JD, Whalley BJ, Baker D, et al. Medicinal Cannabis: Is delta-9-tetrahydrocannabinol necessary for all its effects? J Pharm Pharmacol. 2003;55:1687-1694.

44. Lienau FS, Füllgraf H, Moser A, et al. Why do cannabinoids not show consistent effects as analgetic drugs in multiple sclerosis? Eur J Neurol. 2007;14:1162-1169.
Therapeutics and Clinical Risk Management

\section{Publish your work in this journal}

Therapeutics and Clinical Risk Management is an international, peerreviewed journal of clinical therapeutics and risk management, focusing on concise rapid reporting of clinical studies in all therapeutic areas, outcomes, safety, and programs for the effective, safe, and sustained use of medicines. This journal is indexed on PubMed Central, CAS,

\section{Dovepress}

EMBase, Scopus and the Elsevier Bibliographic databases. The manuscript management system is completely online and includes a very quick and fair peer-review system, which is all easy to use. Visit http://www.dovepress.com/testimonials.php to read real quotes from published authors. 